\title{
A Comparison of the Effects of Medial Prefrontal, Cingulate Cortex, and Cingulum Bundle Lesions on Tests of Spatial Memory: Evidence of a Double Dissociation between Frontal and Cingulum Bundle Contributions
}

\author{
J. P. Aggleton, ${ }^{1}$ N. Neave, ${ }^{1}$ S. Nagle, ${ }^{1}$ and A. Sahgal ${ }^{2}$ \\ ${ }^{1}$ Department of Psychology, University of Durham, Durham, DH1 3LE and ${ }^{2} \mathrm{MRC}$ Neurochemical Pathology Unit, \\ Newcastle General Hospital, Newcastle-Upon-Tyne NE4 6BE, United Kingdom
}

\begin{abstract}
Rats were trained on an automated delayed nonmatchingto-position (DNMP) task. They then received cytotoxic lesions in either the medial prefrontal cortex $(n=13)$ or the cingulate and retrosplenial cortices $(n=8)$, or radiofrequency lesions in either the fornix $(n=6)$ or the cingulum bundle $(n=8)$. Twelve animals served as surgical controls. Only the fornical and medial prefrontal lesions disrupted DNMP performance, both groups showing a loss of accuracy and an increase in bias. The rats were then trained on a lever discrimination and reversal task, the medial prefrontal and fornical groups showing evidence of an increase in bias when compared with the cingulate cortex group. Finally, the rats were trained on a forced alternation task in a T-maze. Marked deficits were observed in the fornix and cingulum bundle groups, but the medial prefrontal and cingulate groups were unimpaired. The double dissociation between the effects of the prefrontal and cingulum bundle lesions highlights the very different nature of the two spatial tasks (DNMP and T-maze alternation), even though both involved a nonmatching rule. These findings may reflect the involvement of divergent outputs from the fornix-anterior thalamic pathway. One possibility is that anterior thalamic projections to the medial prefrontal cortex are concerned with processing egocentric information, while anterior thalamic projections to temporal regions via the cingulum bundle are concerned with allocentric information. The results also indicate that the effects of conventional lesions in the cingulate cortex and medial prefrontal cortex may be compromised by additional damage to the cingulum bundle.
\end{abstract}

[Key words: prefrontal cortex, prelimbic cortex, cingulate cortex, cingulum bundle, fornix, delayed nonmatching-toposition, delayed alternation, spatial memory, rat]

The anterior thalamic nuclei receive numerous direct projections from the hippocampus as well as indirect projections via the mamillary bodies. There is now growing evidence that these

\footnotetext{
Received Apr. 13, 1995; revised June 27, 1995; accepted July 5, 1995.

This research was supported by a grant from the M.R.C. We appreciate the assistance of M. Davies, A. Ennaceur, P. Hunt, S. Lloyd, M. Rolling, and S Whitely.

Correspondence should be addressed to J. P. Aggleton, School of Psychology, University of Wales, College of Cardiff, P.O. Box 901, Cardiff, CF1 3YG, UK.

Copyright $\odot 1995$ Society for Neuroscience $0270-6474 / 95 / 157270-12 \$ 05.00 / 0$
}

connections form part of a hippocampal-fornix-anterior thalamic pathway that is necessary for the normal performance of spatial memory tasks in the rat. One source of evidence has come from studies showing that lesions in the hippocampus, the fornix, and the anterior thalamus all produce severe deficits on spatial tasks such as T-maze alternation, the Morris water maze, and delayed nonmatching-to-position (Aggleton et al., 1986, 1991, 1995; Sutherland and Rodriguez, 1989), while mamillary body lesions disrupt $\mathrm{T}$-maze alternation and water-maze performance (Sutherland and Rodriguez, 1989; Aggleton et al., 1990, 1995). Further support has come from comparisons of brain activity during the performance of memory tasks that require the integrity of the hippocampus (Friedman et al., 1990). The purpose of the present study was to extend our knowledge of this hippocampal-diencephalic system by comparing the effects of lesions in various efferent targets of the anterior thalamic nuclei.

The choice of target area arose from two principal considerations. The first was the presence of direct connections from the anterior thalamic nuclei, the second was evidence that the region might be involved in mnemonic functions similar to those subserved by the hippocampus. The anterior thalamic nuclei are known to project to both the anterior cingulate and retrosplenial cortices, with the projections to the retrosplenial cortex being especially dense (Horikawa et al., 1988; Shibata, 1993). In addition, lesions of this region can disrupt spatial memory tasks such as the Morris water maze (Sutherland et al., 1988; Sutherland and Rodriguez, 1989; Sutherland and Hoesing, 1993) and T-maze alternation (Markowska et al., 1989). There is, however, evidence that damage to the cingulum bundle, which carries projections from the anterior thalamic nuclei to temporal regions, as well as to the cingulate cortices themselves (Domesick, 1970; Mufson and Pandya, 1984), may contribute to these lesion effects (Neave et al., 1994).

The anterior thalamic nuclei have relatively light projections to the medial prefrontal cortices (Conde et al., 1990; Shibata, 1993). These include inputs to the prelimbic region (area 32), which also receives direct hippocampal projections (Jay et al., 1989; Zeng and Steusse, 1991). Lesion studies in rats point to the involvement of the medial prefrontal region in spatial memory tasks taxing allocentric (Thomas and Brito, 1980; Brito et al., 1982; Thomas and Spafford, 1984; Brito and Brito, 1990; Shaw and Aggleton, 1993) and egocentric (Kesner et al., 1989; Dunnett, 1990; King and Corwin, 1992) memory. Some of these studies suggest that the prelimbic cortex is of especial importance (Brito et al., 1982; Brito and Brito, 1990). 
The present study therefore compared cytotoxic lesions in the medial prefrontal cortex (centred in the prelimbic area) with lesions of the cingulate region (anterior cingulate and retrosplenial cortices combined) on spatial tests known to be sensitive to hippocampal and anterior thalamic dysfunction. The study included two further groups, one with cingulum bundle lesions the other with fornix lesions. The former served as a direct comparison with the cingulate cortex lesion group, while the fornix group helped to confirm the sensitivity of the tasks to hippocampal system damage and provide a baseline from which to compare the severity of any other deficits (Aggleton et al., 1992).

\section{Materials and Methods}

The rats were first trained on an automated delayed nonmatching-toposition task (DNMP) to a standard criterion level. Following surgery, the animals were first retested on the DNMP task (Experiment 1) and then trained on two further spatial tasks. The first was a lever discrimination and reversal task run in the same automated apparatus (Experiment 2), the second was a forced-alternation task run in a T-maze (Experiment 3).

\section{Subjects}

The study involved 51 naive male rats of the pigmented DA strain (Bantin and Kingman, Hull, UK). Throughout the period of the experiment the animals were housed individually under diurnal conditions (14 L:10 D), all testing occurring at a regular time during the light period. The animals were fed approximately $15 \mathrm{gm}$ of RM1E laboratory diet (Special Diet Services, Witham, Essex) daily so that they did not drop below $80 \%$ of normal body weight. At the start of testing the animals were aged 4 months and weighed between 205-245 gm. All animals had free access to water.

\section{Apparatus}

Experiments I and 2. The DNMP task and the following discrimination and reversal task were both carried out using seven operant chambers (Campden Instruments Ltd., Loughborough, UK) under the control of two Spider microprocessors (Paul Fray Ltd., Cambridge, UK). Each chamber was fitted with two retractable levers situated $7.5 \mathrm{~cm}$ either side of a central food tray. The magazine tray dispenser, which delivered $45 \mathrm{mg}$ pellets (Campden Instruments), had a hinged Perspex lid at which nose pokes could be recorded. A light was located inside the food tray and a house light was located in the centre of the roof, and a further light was positioned above each lever.

Experiment 3. For the forced-alternation task the animals were tested in a T-maze. The floors of the maze were $10 \mathrm{~cm}$ wide and made of aluminium. The stem was $80 \mathrm{~cm}$ long with a guillotine door located 33 $\mathrm{cm}$ from the beginning. The cross piece was $136 \mathrm{~cm}$ long and at each end there was a food well $4 \mathrm{~cm}$ in diameter and $0.75 \mathrm{~cm}$ deep. The walls of the maze were $17 \mathrm{~cm}$ high and made of clear Perspex. The entire maze was supported on two stands $93 \mathrm{~cm}$ high. Lighting was provided by fluorescent lights suspended $92 \mathrm{~cm}$ above the apparatus, the luminance light levels at the choice point and food wells being 320 and 280 lux, respectively.

\section{Procedure: experiment 1-delayed nonmatching-to-position (DNMP)}

All animals received magazine training followed by an autoshaping pro cedure that trained the rats to respond on both levers (Sahgal, 1983). This was followed by training on the DNMP task to successive criterion stages (Neave et al., 1993). Initially, the rats were trained to respond to a sample lever for a food reward in the central magazine, which was followed immediately by the presentation of both levers ("0"s delay). Responding on the other lever now resulted in food. Iraining continued until the rat reached a criterion of $90 \%$ over 3 consecutive days. The rat was then trained with a $2 \mathrm{sec}$ delay to the same criterion level. This was followed by a fixed number of days with gradually increasing delays between sample lever and test. By the end of this training procedure, all rats were able to respond to a standard sequence of events. After a $10 \mathrm{sec}$ intertrial interval (ITI) either the left or the right lever emerged, and the stimulus light above was illuminated in order to serve as an extra cue. The animal then had to respond to the lever (within 10 sec), upon which the lever was retracted and the magazine tray illuminated. The animal was then required to nose poke at the magazine tray flap so that the first nose poke after a predetermined delay (as in a VI schedule) would cause both levers to emerge and the stimulus lights above them to illuminate. If the animal responded correctly by pressing the lever that had not been the sample (nonmatch) it was then rewarded with one $45 \mathrm{mg}$ food pellet, which was delivered by the central magazine. Either an incorrect response or a failure to respond resulted in a "time-out" of $10 \mathrm{sec}$, during which all of the lights were extinguished and both levers retracted; these were the only occasions when the house light was unlit.

During the initial training procedure there was no retention delay, but following this pretraining period a variable retention delay was placed between the sample and test phases. By requiring the rat to respond on a central panel during this delay it was intended to restrict the use of mediating strategies. The final stages of training consisted of eight sessions, each of 96 trials. The first four of these sessions each contained an equal number of trials with $0,2,4$, and $8 \mathrm{sec}$ retention delays between sample and test. This was followed by two sessions with delays of $0,2,4,8,16$, and $32 \mathrm{sec}$, and a final two sessions with delays of 0 , $4,8,16,32$, and 64 sec.

Following completion of this protocol, the animals received either neurotoxic or radiofrequency lesions placed in either the medial prefrontal cortex $(\mathrm{MPFc})$, the cingulate and retrosplenial cortices $(\mathrm{CINGc})$, the cingulum bundle $(\mathrm{CB})$, or the fornix $(\mathrm{Fx})$. A further group of animals underwent the same initial surgical procedures but did not receive a lesion (SHAM). One animal failed to achieve the preoperative DNMP criteria and was excluded from the rest of the study, while a further three animals died during surgery. The remaining 47 rats used throughout the study were divided as follows: MPFc, $n=13$; CINGc, $n=8$; $\mathrm{CB}, n=8$; Fx, $n=6$; SHAM, $n=12$. Within the MPFe group, a subgroup of animals received slightly smaller lesions that were more specifically targeted at the prelimbic area (PLc, $n=7$ ).

Approximately 6 weeks after surgery all animals were retested on the DNMP task. The first three sessions used only 0-8 sec delays. These were followed by 10 sessions comprising a balanced mixture of 0,2 , $4,8,16$, and $32 \mathrm{sec}$ delays. Each of the postoperative sessions contained 96 trials. Full data analysis was carried out on the 10 sessions with 0 32 s delays.

\section{DNMP: performance measures and analyses}

The data from the $0-32 \mathrm{sec}$ delay condition (postoperative) were analysed to provide three indices of accuracy. These consisted of percent correct, and two measures of sensitivity ( $\mathrm{A}^{\prime}$ and $\mathrm{SI}$ ) derived from nonparametric signal detection models (Frey and Colliver, 1973). Three measures of bias ( $B^{\prime \prime}, \mathrm{RI}$, and Iy) were also calculated. The first ( $\left.\mathrm{B}^{\prime \prime}\right)$ is thought to reflect perceptual bias, that is, the amount of "signalness" in the input required by the subject before responding. The second (RI) is thought to reflect response bias, while the third (Iy) contrasts accuracy between the two levers (Frey and Colliver, 1973; Sahgal, 1987). These signal detection measures can be calculated from the hit $(h)$ and false positive $(f)$ probabilities; in this class of task, $h$ corresponds to the number of correct left responses divided by the total number of correct left plus incorrect right responses, and $f$ corresponds to the number of incorrect left responses divided by the total number of incorrect left plus correct right responses. From this,

$$
\begin{aligned}
\mathrm{A}^{\prime} & =0.5+\left[(h-f)+(h-f)^{2}\right] /\left[4^{*} h^{*}(1-f)\right], \\
\mathrm{SI} & =\left[(h-f] /\left[2 *(h+f)-(h+f)^{2}\right],\right. \\
\mathbf{B}^{\prime \prime} & =\left[\left(h-h^{2}\right)-\left(f-f^{2}\right)\right] /\left[\left(h-h^{2}\right)+\left(f-f^{2}\right)\right], \\
\mathbf{R I} & =[h+f-1] /\left[1-(h-f)^{2}\right],
\end{aligned}
$$

while Iy - (absolute value of left minus right lever corrects)/(total number of corrects).

Finally, estimates of general responsivity were also recorded. These included; (a) latency to respond to the sample lever, (b) latency to make the first magazine response (nose poke), (c) rate of responding to the magazine flap, excluding the 0 s delay condition, (d) choice (and average choice) latencies, and (e) the number of missed trials.

Prior to analysis all data was transformed as appropriate (arcsin, all accuracy and bias indices: logarithmic, all latencies: square-root, misses), and analyzed by parametric analysis of variance (ANOVA). When the $F$-ratios were significant, the means were compared using the Newman-Keuls procedure (Winer, 1971). 


\section{Procedure: Experiment 2-spatial discriminations and reversals}

One week after completing the DNMP task, all animals were trained on a lever discrimination task in the same operant chambers (Neave et al., 1993). For each session, the animal was presented with both levers, one of which had been arbitrarily designated as the correct lever $(\mathbf{S}+)$ and the other as incorrect $(\mathrm{S}-$ ). The levers remained extended until one was pressed (no "miss" trials were permitted) at which point both levers were retracted. Responding on the $\mathrm{S}+$ lever was rewarded with a single $45 \mathrm{mg}$ food pellet, while responding on the $S$ - lever was recorded as an error and no reward presented. Following an intertrial interval of $7 \mathrm{sec}$, the sequence was repeated. Each session consisted of 40 trials, the correct lever always being on the same side throughout a session. All animals received 20 sessions, the position of the correct lever (right or left) following a balanced, pseudorandom sequence.

\section{Procedure: Experiment 3-spatial forced alternation}

Testing began 3 weeks after completion of Experiment 2. Each animal was given 1 or $2 \mathrm{~d}$ of pretraining in order to run reliably down the stem of the maze to find food pellets in the food wells in both arms. Following this, the experiment began. At the start of each trial, which consisted of two stages, three food pellets (45 mg Campden Instruments, Loughborough), were placed in each food well and a wooden block was placed at the neck of the T-maze so closing off one arm. As a consequence, on each 'sample run' the animal was forced to enter the open arm where it was allowed to eat the food there. The animal was then picked up and confined in the start box for a delay of $15 \mathrm{sec}$, during which the wooden block was removed. The door to the start box was then opened and the animal allowed a free choice between the two arms of the T-maze. On the 'choice run' the animal was deemed to have chosen when it had placed a back foot in one of the two arms, no retracing was permitted. If the rat had alternated, i.e., had entered the arm not previously visited on the 'sample run,' it was allowed to eat the food reward (three pellets) before being returned to its cage. If the other arm was chosen, i.e., the same arm as visited on the 'sample run,' the rat was confined to that arm for approximately $10 \mathrm{sec}$, and then returned to its cage.

The rats were tested in groups of four with each rat having one trial in turn, so that the intertrial interval was about $4 \mathrm{~min}$. The animals received six trials a day, for a total of six sessions. This was immediately followed by a further 10 sessions in which the six daily trials were divided equally between those with retention delays of either 10,30, or $60 \mathrm{sec}$, making a total of 20 trials at each of the three delays. The retention delay corresponded to the time the animal spent in the start box prior to the "choice run."

\section{Surgical and histological procedures}

Each animal was anaesthetized by intraperitoneal injection $(6 \mathrm{ml} / \mathrm{kg})$ of Sagatal. The animal was then placed in a stereotaxic headholder (David Kopf Instruments, Tujunga, CA), and the scalp retracted to expose the skull. A craniotomy was then made above the sagittal sinus and the dura cut to expose the cortex above the appropriate region.

For the larger of the medial prefrontal cortex lesions (MPFc), injections of $0.28 \mu \mathrm{l}$ of $0.09 \mathrm{M} \mathrm{N}$-methyl-D-aspartic acid (NMDA) (Sigma Chemical Company Ltd., Poole, UK) dissolved in phosphate buffer ( $\mathrm{pH}$ 7.2) were delivered through a $I \mu l$ Hamilton syringe into two different sites in each of two different vertical tracts. This resulted in a total of four injection sites in each hemisphere. The stereotaxic coordinates relative to ear-bar zero, with the incisor bar set at +5.0 relative to the horizontal plane, were as follows: AP +8.7 , LAT \pm 0.7 , with depths of $4.5 \mathrm{~mm}$ and $2.2 \mathrm{~mm}$ below the top of the cortex, and AP +10.0, LAT \pm 0.7 , with depths of $3.5 \mathrm{~mm}$ and $2.0 \mathrm{~mm}$ below the top of cortex. Each injection was made gradually over a $4 \mathrm{~min}$ period and the needle allowed to remain in situ for a further $4 \mathrm{~min}$ before being withdrawn. After completion of the surgical procedure, sulphanilamide powder was applied and the skin sutured.

The same procedure was used for the more restricted prelimbic lesions (PLc) except that only a single injection was made in each of the two tracts, and a total of $0.3 \mu \mathrm{l}$ of $0.09 \mathrm{M}$ NMDA was injected in each of these sites. The coordinates relative to ear-bar zero were: $\mathrm{AP}+8.7$, LAT \pm 0.7 , depth from top of cortex $3.5 \mathrm{~mm}$, and AP +10.0, LAT \pm 0.7 , depth from top of cortex $2.8 \mathrm{~mm}$.

For the total cingulate cortex lesions (CINGc) injections of $0.3 \mu \mathrm{l}$ of $0.09 \mathrm{~m}$ NMDA were injected into five sites per hemisphere. The co- ordinates of these injections relative to ear-bar zero were, going rostral to caudal: $\mathrm{AP}+7.6, \mathrm{LAT} \pm 0.7 ; \mathrm{AP}+6.0, \mathrm{LAT} \pm 0.8 ; \mathrm{AP}+4.3, \mathrm{LAT}$ $\pm 0.8 ; \mathrm{AP}+2.6$, LAT $\pm 0.8 ; \mathrm{AP}+0.9$, LAT \pm 0.8 . The depth at each sitc, going rostral to caudal, was $2.0 \mathrm{~mm}, 2.0 \mathrm{~mm}, 1.7 \mathrm{~mm}, 1.1 \mathrm{~mm}$, and $2.2 \mathrm{~mm}$ from the top of cortex.

The initial stages of the cingulum bundle surgeries (CB) were the same as those described for the animals with cortical lesions, but the actual CB lesions were made by radiofrequency. A Radionics TCZ (Radionics Inc., Burlington) electrode $(0.3 \mathrm{~mm}$ tip length and $0.25 \mathrm{~mm}$ diameter) was lowered vertically into three sites per hemisphere and the tip temperature raised to $75^{\circ} \mathrm{C}$ for $60 \mathrm{sec}$ using an RFG4-A Lesion Maker (Radionics Inc.. Burlington). The coordinates of these lesions, relative to ear-bar zero were as follows: $\mathrm{AP}+7.9, \mathrm{LAT} \pm 1.1, \mathrm{AP}+3.7$, $\mathrm{LAT} \pm 1.0$, and AP +1.4 , LAT \pm 0.9 . The depth at the three sites, going from rostral to caudal, were $2.1 \mathrm{~mm}, 1.7 \mathrm{~mm}$, and $1.9 \mathrm{~mm}$ below the top of cortex. In five of the eight $\mathrm{CB}$ animals a further lesion $(60 \mathrm{sec}$, $75^{\circ} \mathrm{C}$ ) was placed $0.4 \mathrm{~mm}$ dorsal to each of those already described (i.e., these animals received two lesions in each of the three tracts per hemisphere).

The animals in the fornix ( $F x)$ group underwent the same procedure as the CB group with only two radiofrequency lesions $\left(60 \mathrm{sec}, 75^{\circ} \mathrm{C}\right)$ being made in each hemisphere. The stereotaxic coordinates of the lesions relative to ear-bar zero were: AP +5.3 , LAT \pm 0.7, HT $3.7 \mathrm{~mm}$ below the top of cortex, and AP +5.3, LAT \pm 1.7 , HT $3.8 \mathrm{~mm}$ below the top of cortex. Finally, the animals acting as surgical controls (SHAM) received the same initial treatment as the animals receiving prefrontal lesions, but although the dura was cut and retracted no injection of NMDA was made.

On completion of the experiment the animals were killed and perfused intracardially with $5 \%$ formol saline. The brains were then rapidly removed and placed in 5\% formol saline. Subsequently, the brains were blocked, cmbedded in wax (Paraplast), and cut into $10 \mu \mathrm{m}$ coronal sections. Every tenth section was mounted and stained with cresyl violet, a Nissl stain.

\section{Results}

\section{Histological analysis}

The cytoarchitectonic borders and nomenclature were taken from the atlas by Swanson (1992). Following histological analysis, none of the animals were discarded from their respective surgical groups. Of the MPFc group, the subgroup of six animals with the larger NMDA injections in the medial prefrontal cortex all showed extensive lesions centred in the prelimbic region (Figs. 1 and 2). These lesions extended from just behind the rostral pole to the level of the genu of the corpus callosum. In all cases there was very extensive bilateral damage to the prelimbic region (area 32), and in all but one animal this extended ventrally to involve much of the infralimbic area. The lesions also extended dorsally to include the more rostral and ventral parts of the anterior cingulate area ACAd, but in three cases the involvement of ACAd was very slight. The induseum griseum was involved in all cases, and in two cases the lesion extended caudally to involve the very rostral limit of the septum. The nucleus accumbens and the olfactory bulbs were spared. The largest and the smallest of these medial prefrontal lesions are depicted in Figure 1.

The lesions in the seven animals receiving more restricted injections of NMDA in the prefrontal cortex (PLc) were again centred in the prelimbic region, but less extensive in total area (Figs. 1 and 2). In all but two cases the lesions began at the pole, and they continued rostrally to a level just in front of the genu. In all cases there was considerable bilateral cell loss in the prelimbic area, only the most caudal regions being spared. The infralimbic cortex was extensively damaged in most cases, but in two animals it was largely spared. Damage to the cingulate cortex was typically restricted to the most rostral and ventral portion of ACAd. The induseum griseum was spared in all cases. 

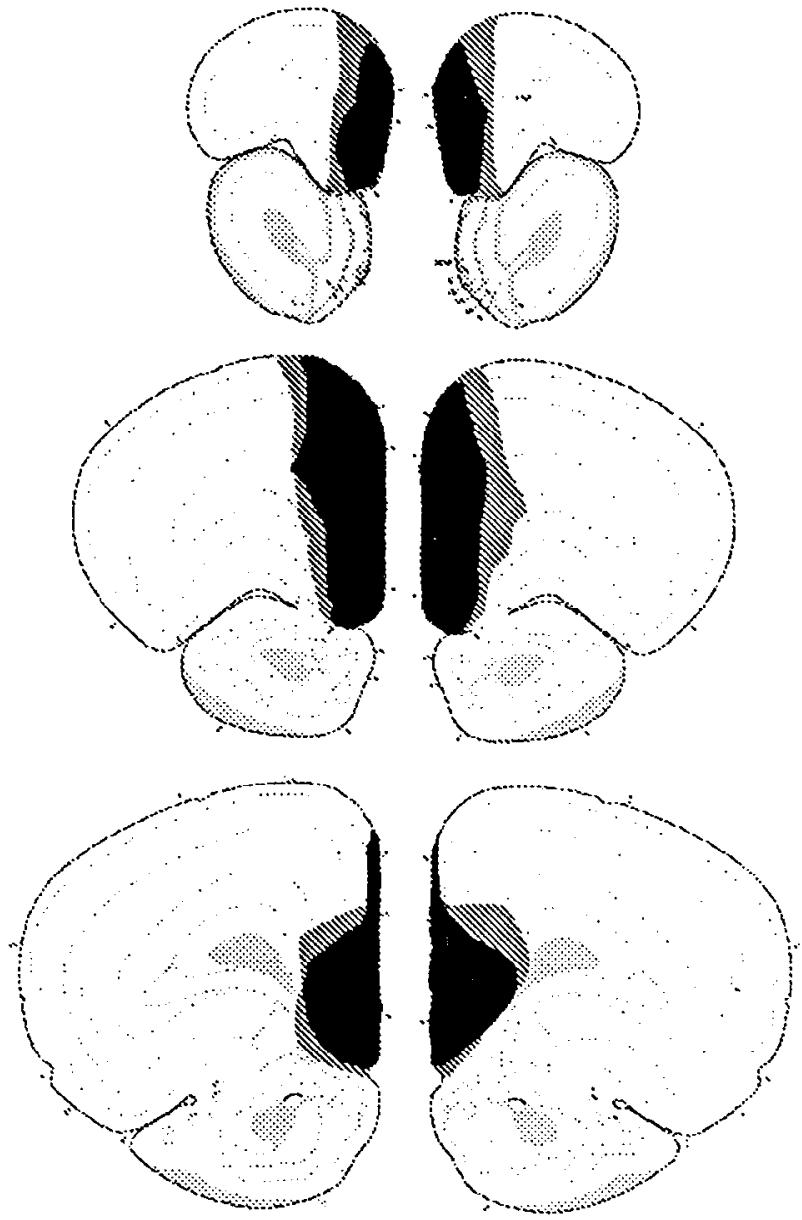

\section{2}
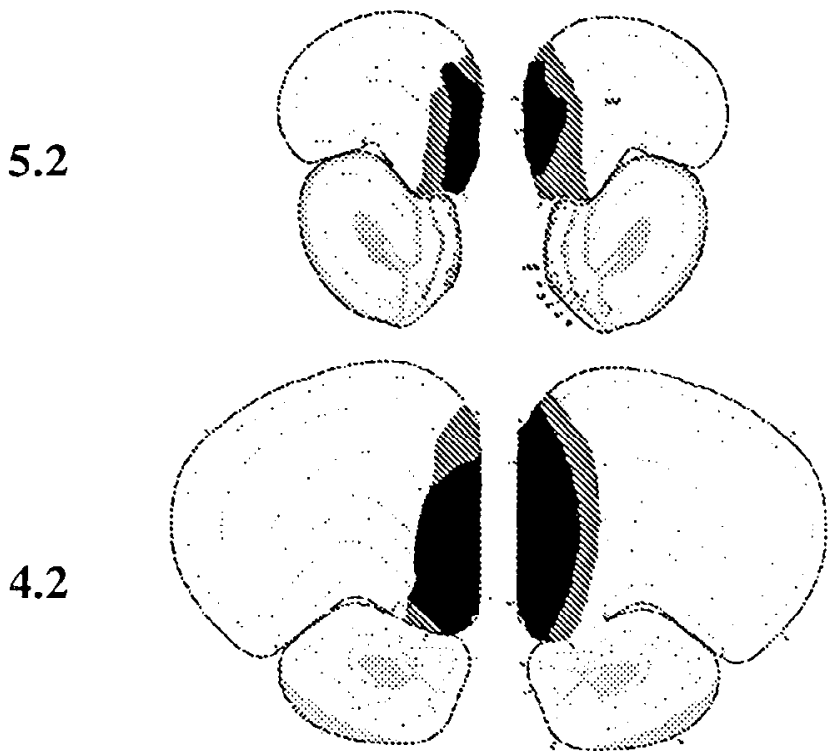

3.2
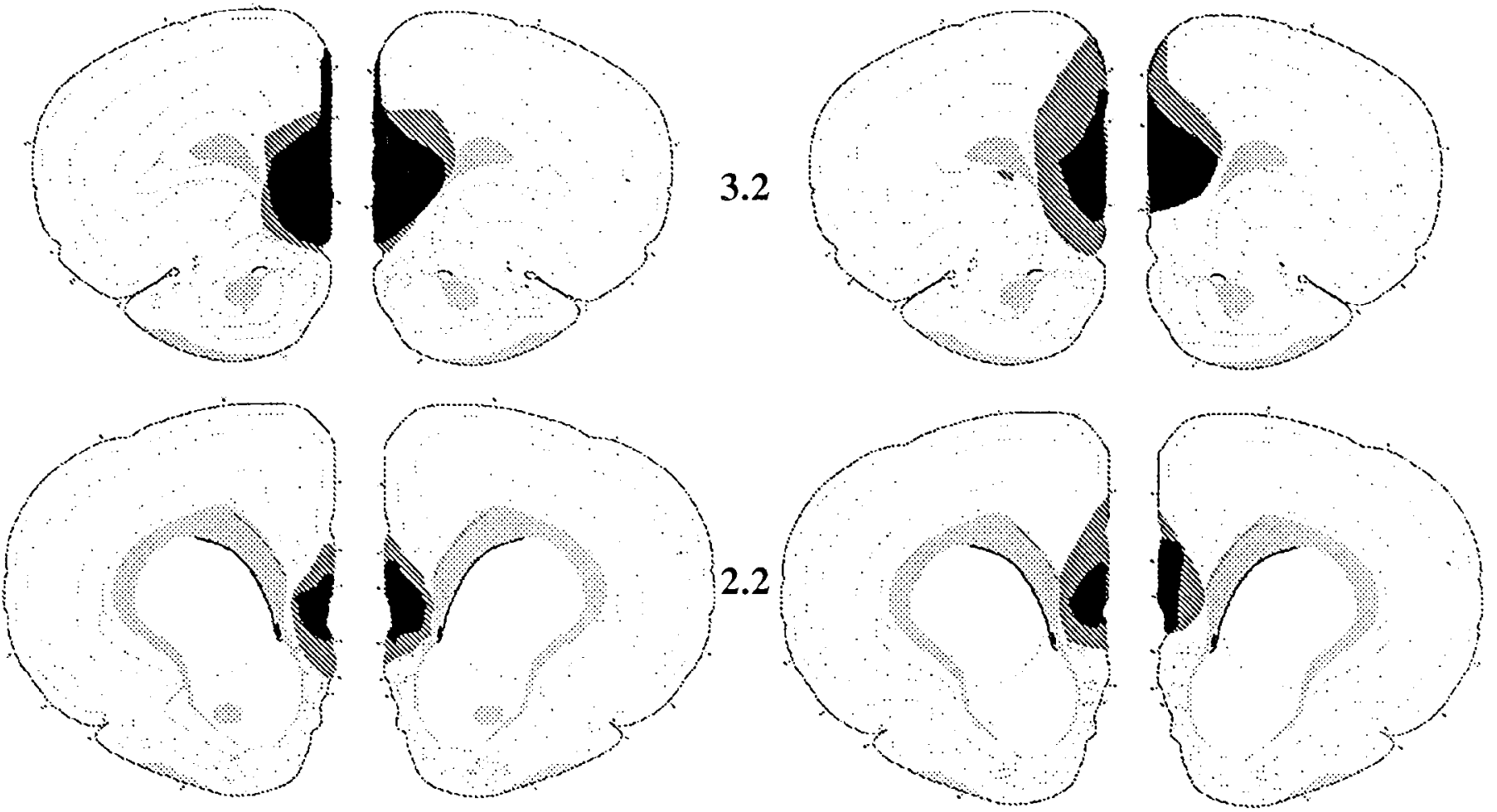

MPFc

\section{MPFc}

(PLc)

Figure 1. Series of coronal sections showing the extent of the largest (diagonal lines) and smallest (black) of the medial prefrontal lesions ( $M P F c$ ). The left-hand column showns the MPFc animals that received four NMDA injections per hemisphere, the right-hand column shows the animals receiving two injections per hemisphere $(P L C)$. The sections are modified from Swanson (1992), and the numbers correspond to their AP position relative to bregma.

The lesions in the CINGc group consistently involved the entire extent of the cingulate (anterior cingulate and retrosplenial) cortices that lie dorsal to the corpus callosum (Figs. 2 and 3). The cell loss in the anterior cingulate cortex began at the genu and continued caudally to the retrosplenial cortex. The lesions extended dorsally to include ACAv, ACAd, and parts of the adjacent secondary motor area (Fig. 3). The retrosplenial dam- age was continuous and extended caudally up to and beyond the splenium. Throughout the level of the corpus callosum both the ventral and the dorsal parts of the retrosplenial cortex showed an almost complete loss of cells (Fig. 4). Caudal to the splenium there was often sparing of the dorsal retrosplenial cortex, but the involvement of the ventral retrosplenial cortex often continued to near its caudal limit (Fig. 2). Some cellular loss was 


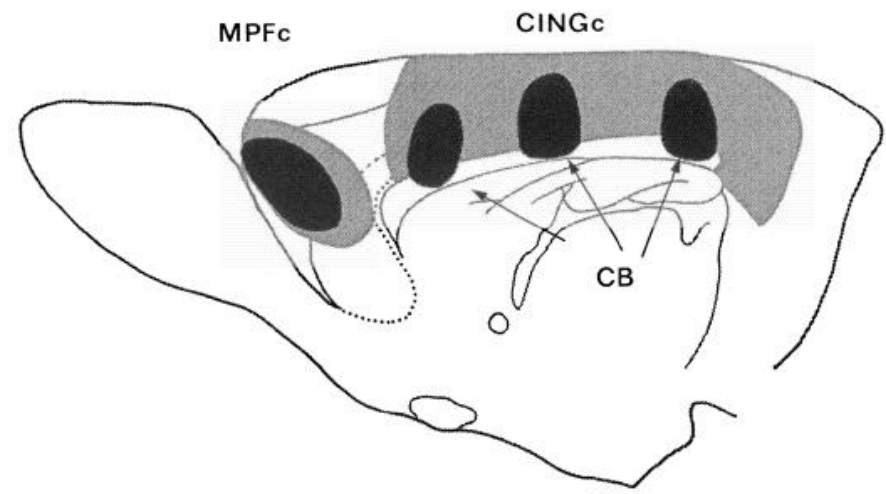

Figure 2. View of medial surface of rat brain showing the extent of typical examples of the cingulate cortex lesion (CINGc, gray), cinglum bundle lesion $(C B$, black), and the medial prefrontal lesions $(M P F C)$. The larger MPFc lesions are shown in gray, while the typical size of the smaller Plc lesions is shown in black.

observed in those parts of the hippocampal field CA1 in closest proximity to the ventral retrosplenial cortex (Fig. 3). In all cases there was evidence of cellular degeneration in the anterior ventral thalamic nucleus, although the cingulum bundle appeared to be intact.
The cingulum bundle surgeries (CB) involved three bilateral radiofrequency lesions placed at different AP levels. In all $\mathrm{CB}$ cases there was very considerable, bilateral damage to the bundle, at more than one level (Figs. 3 and 5). Consistent with this there was very marked cellular loss in the anterior ventral thalamic nucleus, which sometimes extended into the most lateral part of the anterior medial nucleus. The lesions did not involve the fornix, but in four of the eight cases there was very minor damage to the dorsal limit of the hippocampus (principally to the alveus) in a very restricted anterior-posterior portion below the mid AP lesion. In three of these four cases the hippocampal damage was only unilateral. The extent of damage to those parts of the anterior cingulate and retrosplenial cortices adjacent to the cingulum bundle sites was variable, but in the majority of cases the lesions were quite selective, with limited direct cortical damage (Figs. 3 and 5). Even in those cases with the greatest cingulate cortex damage (Fig. 3) the extent of the lesion at the level of the radiofrequency probe was less than that in the NMDA cases (CINGc), and there were always regions of intact cortex between the three radiofrequency lesion sites.

In three of the six fornix lesions (Fx) the tract was completely transected. In two of the remaining animals the fimbria/fornix was completely cut in one hemisphere, and only the most lateral tips of the fimbria were spared. In the case with the smallest
1.2

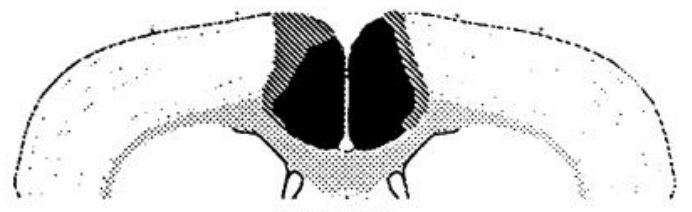

CINGc

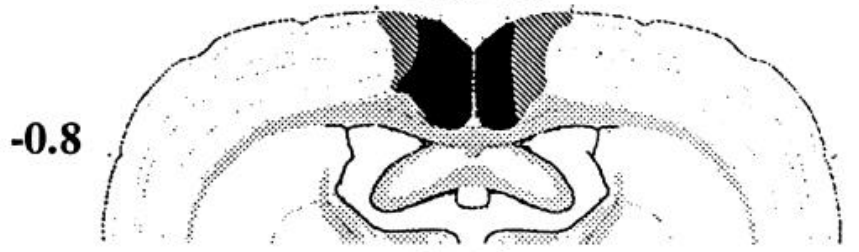

CINGc

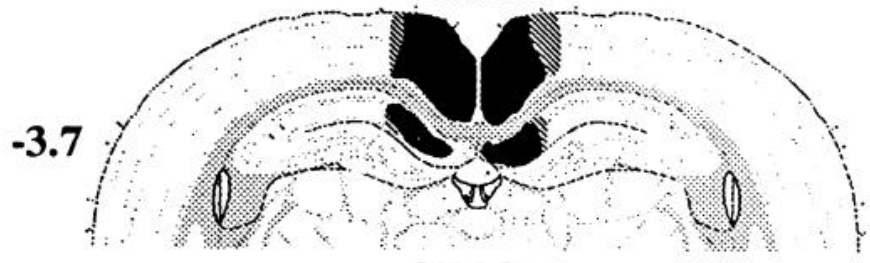

CINGc

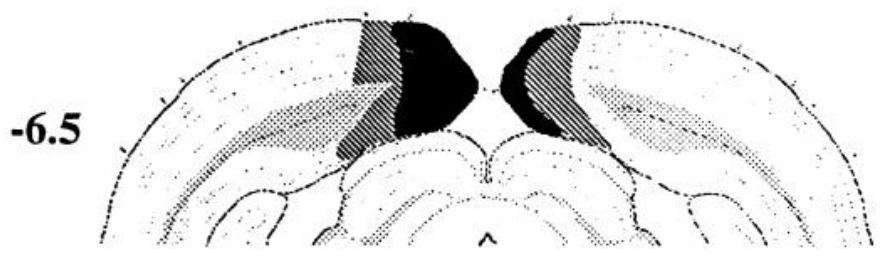

CINGc

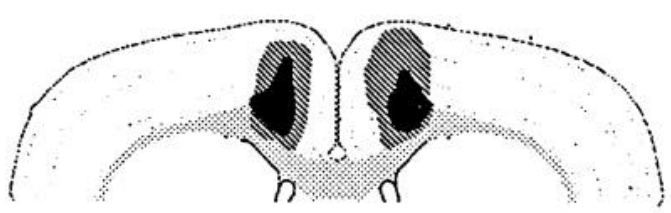

1.2

CB

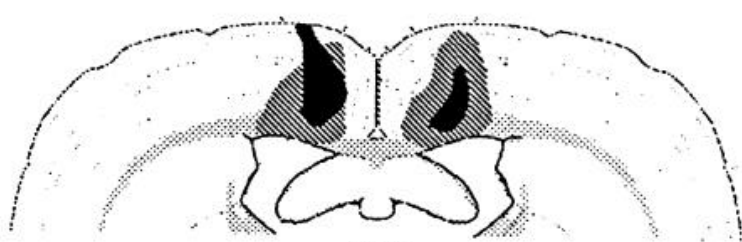

$-0.8$

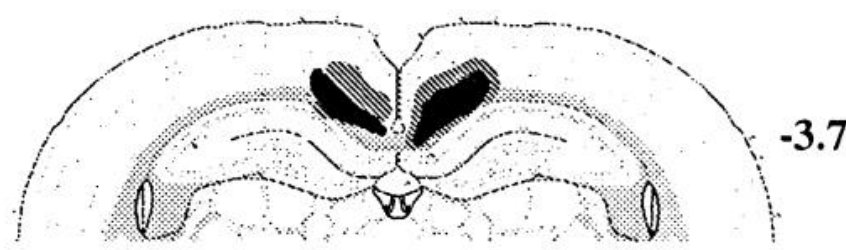

CB

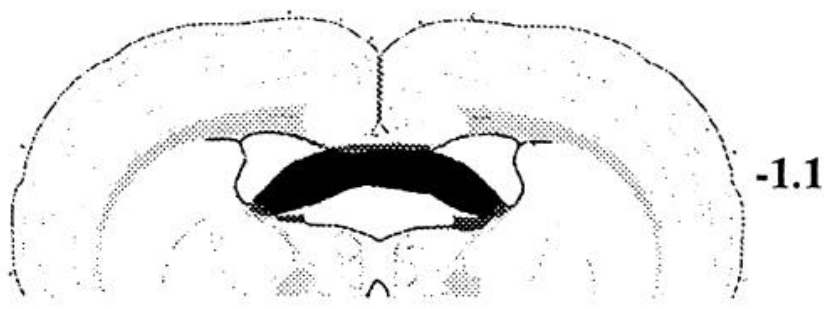

FX

Figure 3. Series of coronal sections showing the extent of the largest (diagonal lines) and smallest (black) of the cingulate cortex (CINGc), cingulum bundle $(C B)$, and fornix $(F x)$ lesions. The sections are modified from Swanson (1992), and the numbers correspond to their AP position relative to bregma. 

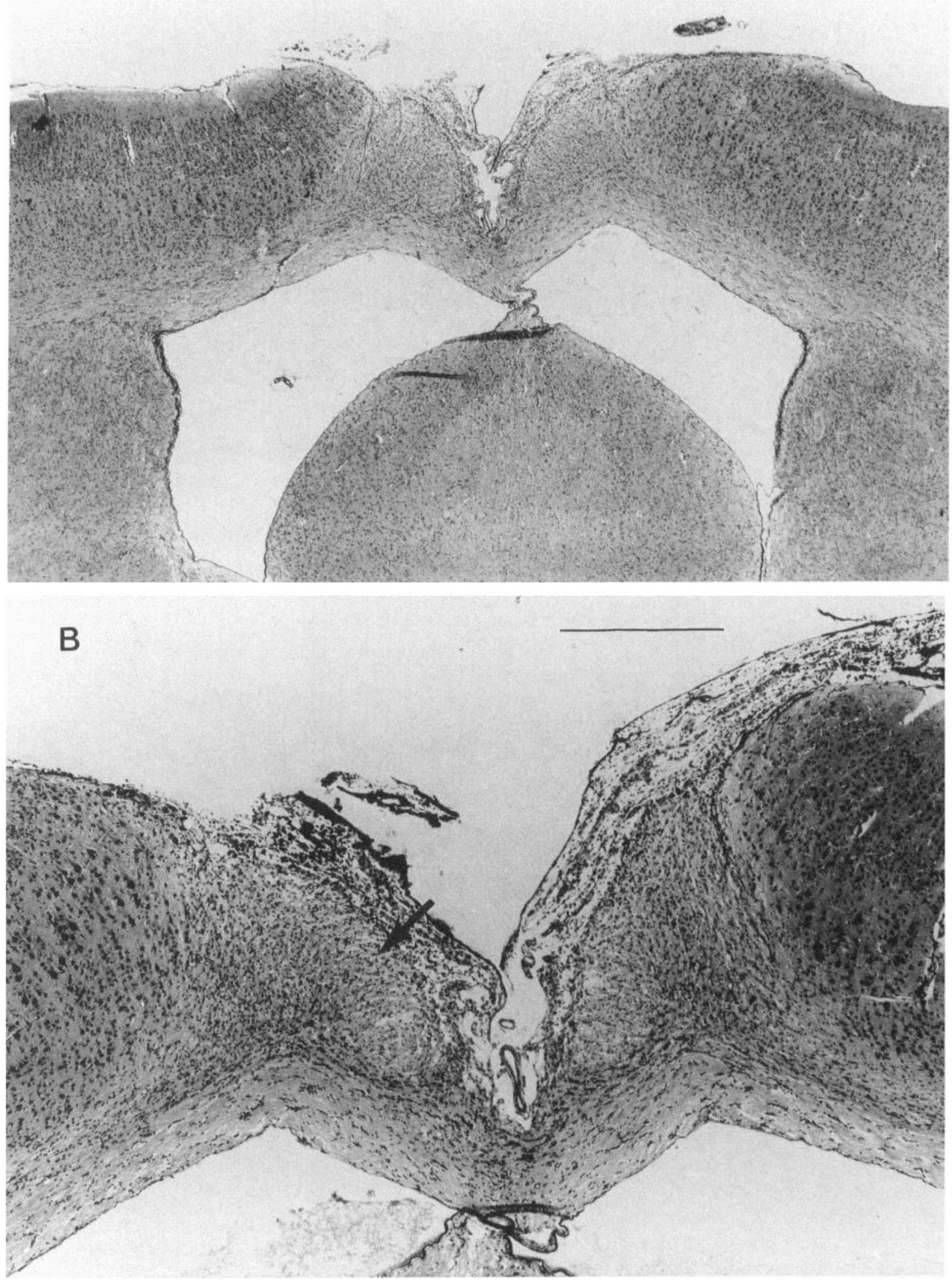

Figure 4. Photomicrographs of coronal sections (Nissl stain) showing the appearance of the cytotoxic cingulate cortex lesions (CINGc) at two different magnifications. The arrow in $B$ points to the cingulum bundle, which appears spared in contrast to the overlying cortices. The scale bars depict $500 \mu \mathrm{m}$. 

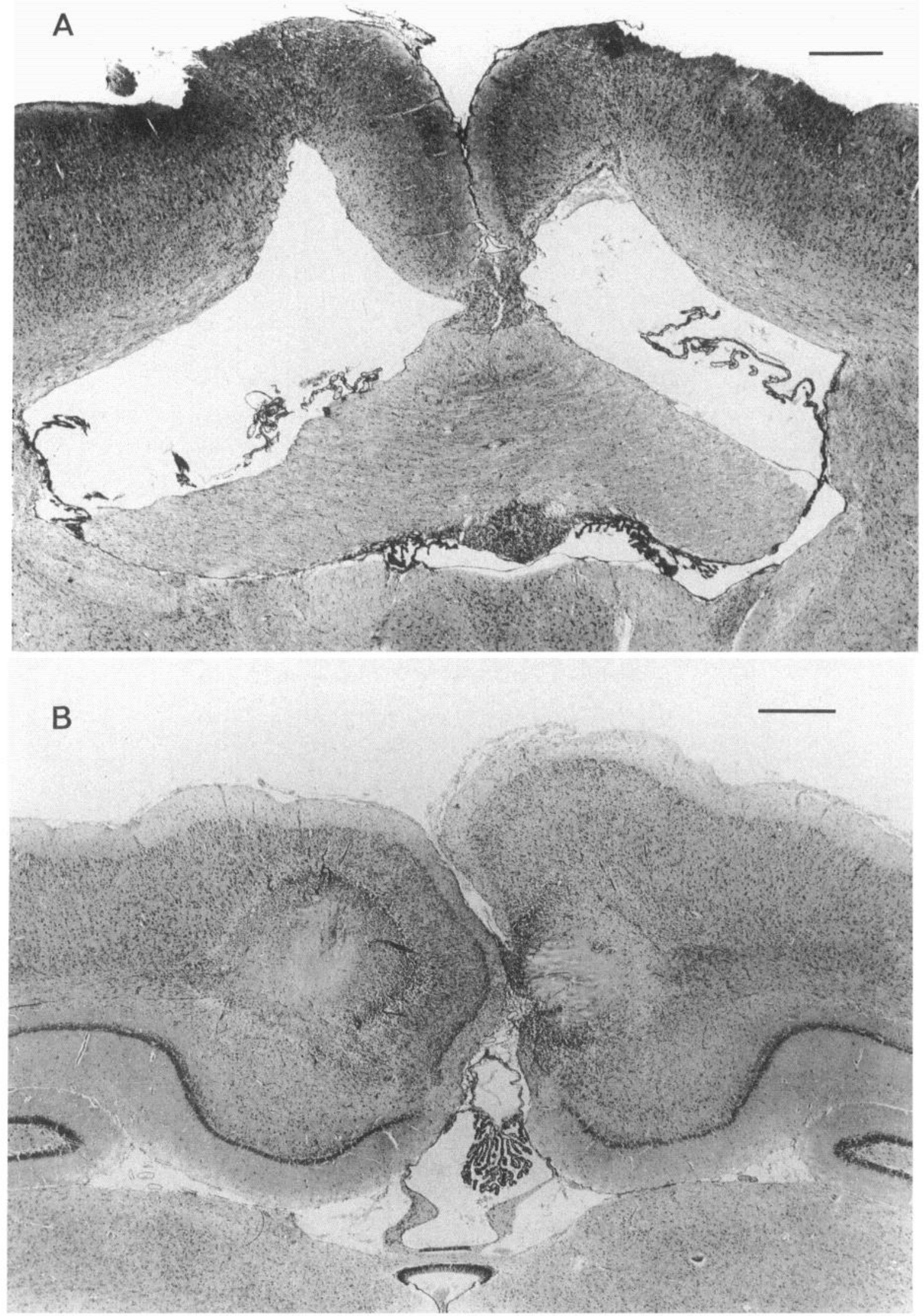

Figure 5. Photomicrographs of coronal sections (Nissl stain) showing the appearance of the radiofrequency cingulum bundle ( $C B$ ) lesions at mid $(A)$ and caudal $(B)$ levels. The scale bars depict $500 \mu \mathrm{m}$. 


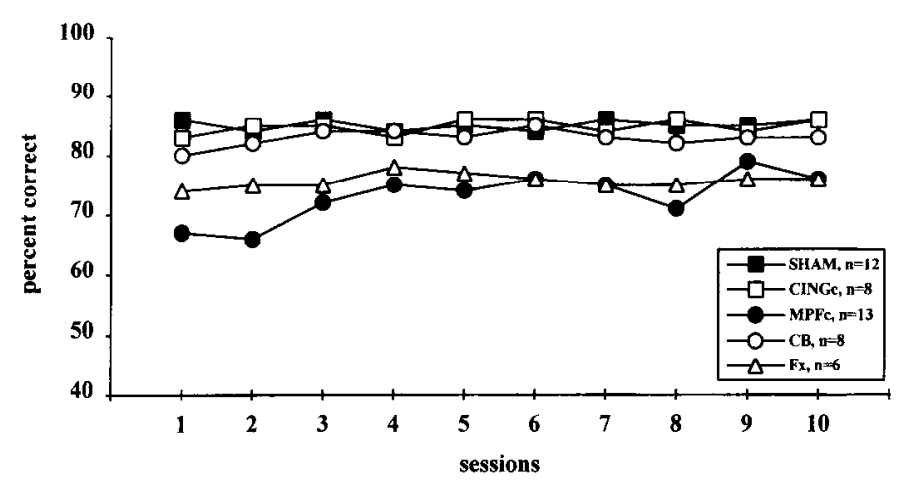

Figure 6. Mean percentage correct scores on the DNMP task over each of the 10 postoperative sessions using the pooled results from the six delay conditions $(0-32 \mathrm{sec})$.

lesion the most lateral tips of the fimbria were spared bilaterally (Fig. 3). In four of the Fx cases there was very slight involvement of the most dorsal limits of the anterior ventral and anterior dorsal thalamic nuclei.

\section{Comparisons among animals with medial prefrontal lesions} $M P F C$

Within the group of 13 rats with prefrontal lesions, a subset of seven animals received slightly more discrete lesions targeted at the prelimbic area. In view of the extent of overlap between the two sets of lesions, a series of initial analyses compared these two subgroups in order to determine if they should be combined prior to subsequent comparisons. For the DNMP task it was found that the mean percentage correct scores of the two subgroups over the 10 postoperative sessions (0-32 sec delays) were very similar $(75.3 \%$ for the larger lesions and $73.1 \%$ for the smaller "prelimbic" cases) and did not differ $(t<1)$. Furthermore, a series of statistical comparisons involving these two groups failed to find any evidence of a significant difference in DNMP performance as measured by any of the accuracy, bias, or responsivity indices. Of the comparisons used in Experiment 2 and 3 only one, performance on the first two sessions of the lever discrimination found any evidence of a group difference $[t(11)=2.26, p<0.05]$. In view of the fact that only one out of a total of 17 comparisons revealed a difference at the 0.05 level, it was decided to treat the animals as a single group (MPFc) for all of the subsequent analyses. Consistent with this, the large majority of the scores of the two subgroups were extremcly similar.

\section{Experiment 1: DNMP}

Comparisons of postoperative DNMP performance were based on the final 10 daily sessions, i.e., sessions of 96 trials each containing a counterbalanced sequence of $0,2,4,8,16$, and 32 sec delays. Prior to these analyses, the preoperative scores of the five experimental groups were compared in order to test whether the groups were appropriately matched. A comparison based on the number of training sessions required to reach criterion performance (from initial training to $2 \mathrm{sec}$ delay) failed to find any evidence of a difference between the five groups ( $F$ $<1)$. Similarly, there was no group difference when the mean scores from the last four preoperative DNMP sessions $(0-32 \mathrm{sec}$ and 0-64 sec combined) were compared $(F<1)$.

$D N M P$ accuracy measures. An analysis of variance using the pcrcent correct scores (Figs. 6 and 7) over the 10 postoperative
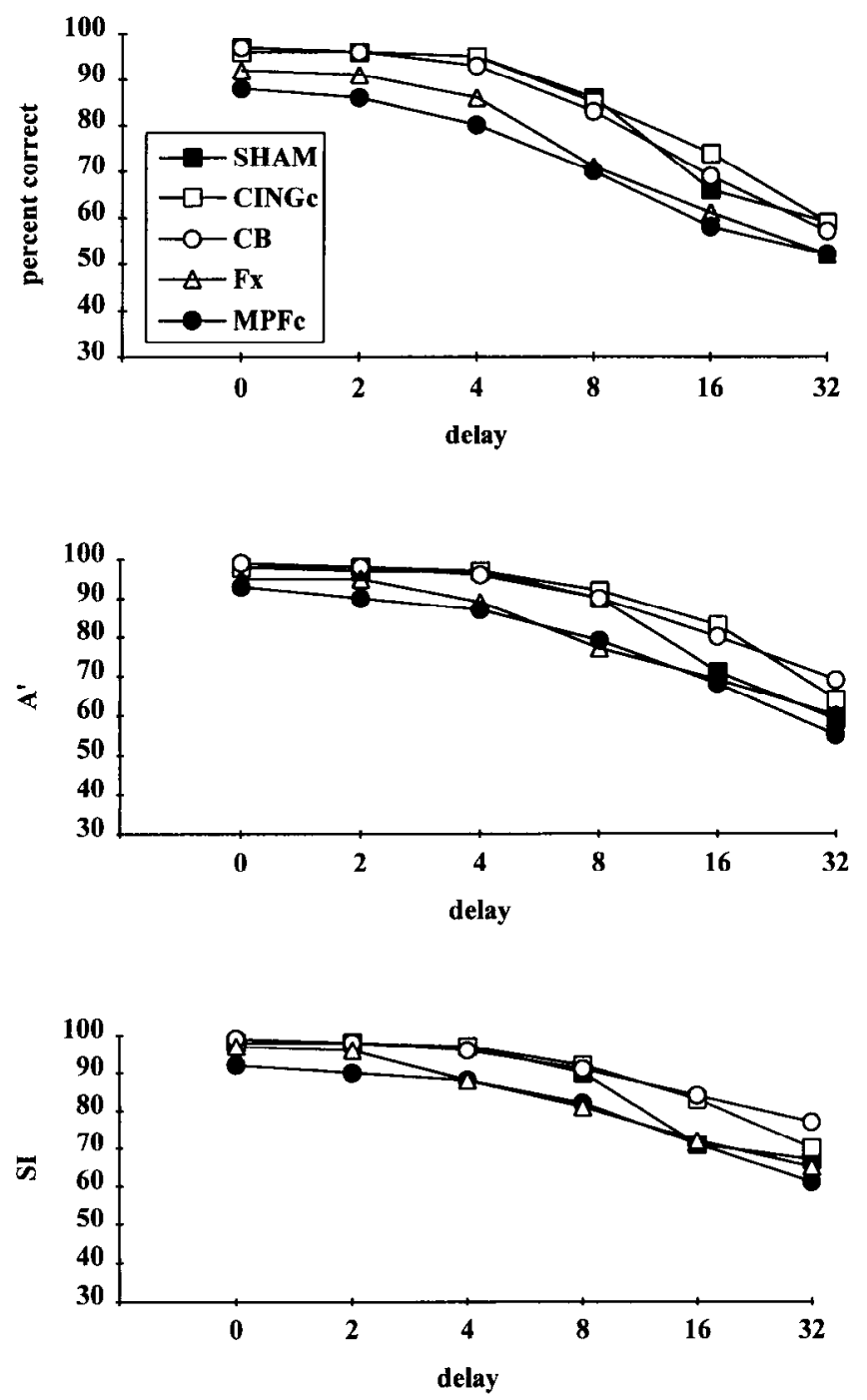

Figure 7. Mean scores on accuracy indices (percentage correct, $A^{\prime}$, SI) over the 10 DNMP sessions with 0-32 sec delays. See text for explanation of indices. All scores have been converted to absolute values and read from 0 to 100 .

sessions revealed a significant group effect $[F(4,43)=9.18, p$ $<0.01]$, a significant effect of delay $[F(5,215)=521.3, p<$ $0.01]$, and a group $\times$ delay interaction $[F(20,215)=2.35, p<$ $0.01]$. Subsequent Newman-Keuls tests revealed that the group difference reflected the poorer performances of both the Fx and MPFc groups, who differed from all of the other groups $(p<$ 0.05 ) but not from one another. There were no other group differences. The group $\times$ delay interaction reflected the unusually steep decline in scores by the Fx group, while the MPFc group did not show an abnormally steep forgetting curve. These two groups also showed a deficit when compared with the SHAM animals on just the $0 \mathrm{sec}$ delay scores $(p<0.05)$. There was also an effect of session $[F(9,387)=2.38, p<0.05]$, as the animals improved with additional training.

A similar pattern of results was found for the two measures of sensitivity derived from signal detection theory, $\mathrm{A}^{\prime}$ and SI (Fig. 7). The $A^{\prime}$ measure revealed highly significant effects of group $[F(4,43)=7.88, p<0.01]$, session $[F(9,387)=1.92, p$ $<0.05]$, and delay $[F(5,215(=323.7, p<0.01]$, as well as a group $\times$ delay interaction $[F(20,215)=1.84, p<0.05]$. As 

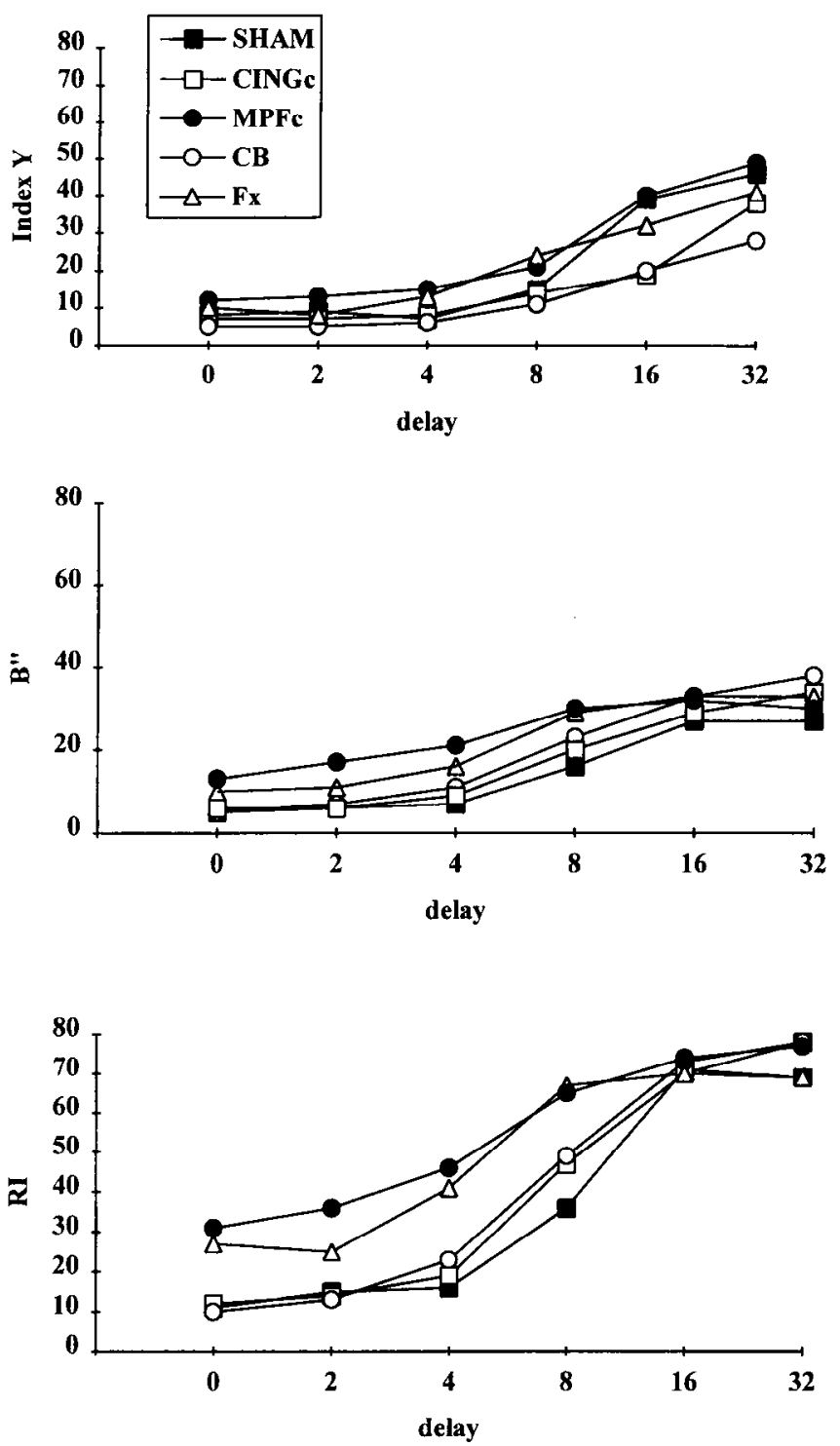

Figure 8. Mean scores on three bias indices $\left(R I, B^{\prime \prime}, I y\right)$ over the 10 DNMP sessions with $0-32 \mathrm{sec}$ delay. All scores have been converted to absolute values. A score of 100 represents a complete bias, while 0 is neutral, or no bias.

before, the group effects reflected the abnormal behaviour of the Fx and MPFc groups who differed from the other groups $(p<$ 0.05 ) but not from each other. The group by delay interaction reflected the different performance profiles of the SHAM and Fx groups (Fig. 7).

The SI measure only revealed significant effects of group $[F(4,43)=6.00, p<0.01]$, and delay $[F(5,215)=133.25, p$ $<0.011$. The group effect was the result of the Fx and MPFc groups differing from both the CINGc and the CB groups $(p<$ 0.05 ). For this measure the difference between Fx and SHAM groups was not significant (Fig. 7), but the MPFc animals did perform at a lower level than the SHAM animals $(p<0.05)$.

DNMP bias indices. As expected from previous studies, all three bias indices (Iy, $\mathrm{B}^{\prime \prime}$, and $\mathrm{RI}$ ) demonstrated delay effects ( $p$ $<0.01$ in all cases; Fig. 8). Each bias index also revealed a significant group effect [ly, $F(4,43)=5.55, p<0.01 ; \mathrm{B}^{\prime \prime}$, $F(4,43)=8.84, p<0.01 ; \mathrm{RI}, F(4,43)=9.86, p<0.01]$, and for two of the indices there was also a significant group $\times$ delay

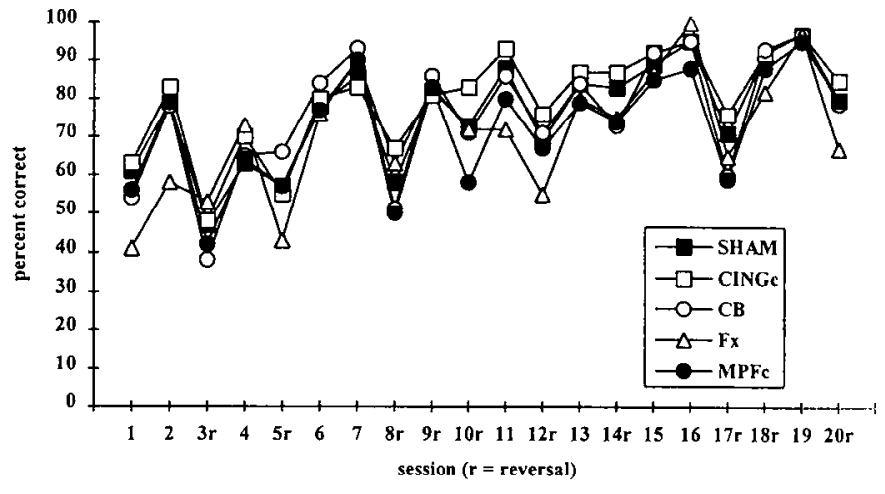

Figure 9. Mean percentage correct scores for the CB, CINGc, MPFc, Fx, and SHAM groups on the lever discrimination and reversal task over the 20 sessions. Sessions where the correct lever changed from the preceeding session ("reversal") are labeled as $R$.

interaction $\left[\mathrm{B}^{\prime \prime}, F(20,215)=2.92, p<0.01 ; \mathrm{RI}, F(20,215)=\right.$ $2.69, p<0.01]$. It can be seen that both the MPFc and the Fx groups showed abnormally high levels of bias at the shortest delays, but by the longest delays these group differences had often disappeared (Fig. 8). The group by delay interactions for $B^{\prime \prime}$ and RI reflected the unusual bias scores of the MPFc group. In addition, both $\mathrm{B}^{\prime \prime}$ and RI exhibited an effect of session $(p<$ 0.01 in both cases) as the bias decreased with practice. Finally, in the case of index Iy there was a significant session $\times$ delay interaction $[F(45,1935)-1.41, p<0.05]$ as the eflect of delay lessened with further practice.

For index RI, both the MPFc and Fx animals showed higher levels of bias than the other three groups, with no other differences being revealed (Fig. 8). In the case of ly, a slightly different pattern emerged. This was because the MPFc and Fx groups both displayed higher levels of bias than the CB and the CINGc groups, but they did not differ from the SHAM group. For index B", both the MPFc and Fx groups had higher bias scores than both the SH $\wedge$ M and the CINGc groups, but only MPFc group differed from the CB animals.

DNMP general responsivity. There were no group effects for a number of general measures of responsivity. These were: total misses $[F(4,43)=1.97, p>0.05]$, magazine responses (nose pokes) $(F<1)$, and average choice latency $[F(4,43)=1.70, p$ $>0.05]$. Group differences were found, however, for mean latency of first magazine response $[F(4,43)=6.60, p<0.01]$, and for the mean latency to sample $[F(4,43)=3.11, p<0.05]$. The first group effect reflected the slower latencies of both CB and MPFc groups who differed from the other three groups; the second group difference (mean latency to sample) was due to the SHAM animals being quicker than all the other groups, while the CB group were slower than the Fx and MPFc groups, as well as the SHAM animals.

\section{Experiment 2: Spatial discriminations and reversals}

The second experiment used a spatial (lever) discrimination task (sessions 1 and 2 ) followed by a series of reversals between specified sessions (Fig. 9). Although there was no group effect over the first two discrimination sessions $[F(4,46)=2.36, p>$ $0.05]$, a significant group effect was found for the remaining 18 sessions $[F(4,46)=2.69, p<0.05]$. Subsequent NewmanKeuls tests revealed that the only group difference was between the MPFc and CINGc groups, who respectively obtained the 


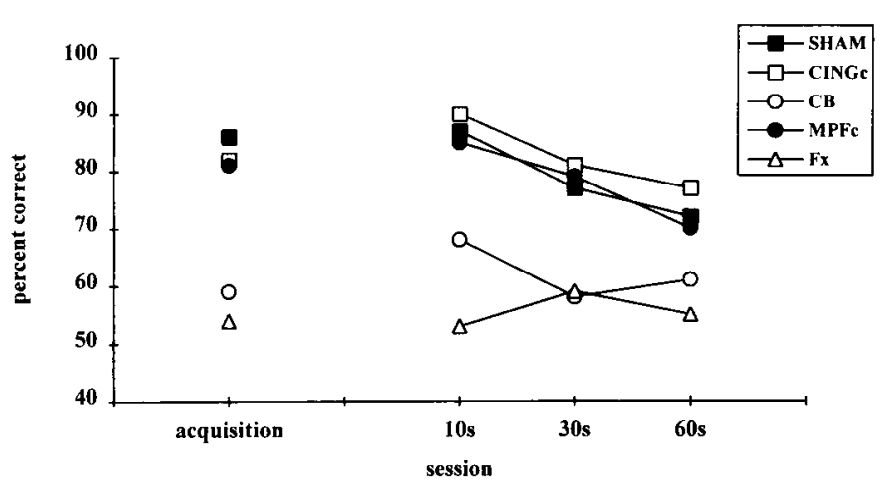

Figure 10. Spatial forced alternation in a T-maze. The graph shows the mean scores of each group over the initial six acquisition sessions, and the mean scores of the same groups when tested over each of three retention intervals.

lowest and the highest overall scores on these 18 sessions. No other group differences were found.

By excluding the first session, the remaining 19 sessions could be divided into those nine sessions in which the correct lever was in the same position as it had been on the previous session ("consistent"), and those 10 sessions in which the correct lever reversed side between sessions ("reversal"). Analyses of these two sets of scores confirmed that all groups performed worse on the 'reversal' sessions $[F(1,42)=262.05, p<0.001]$, but that there was also a significant group effect $[F(4,42)=3.11, p$ $<0.05$ ]. Subsequent Newman-Keuls tests showed that the group differences once again reflected the poor scores of the MPFc and Fx groups as compared with the CINGc group $(p<$ 0.05 ). There were no other group differences and no group $\times$ type of session interaction, even though the performance of the MPFc and Fx groups appeared poorer on the "reversal" sessions. A similar pattern of results was found when only the first 10 trials of each session, when the effects of the previous session might be expected to be most apparent, were considered.

\section{Experiment 3: spatial forced alternation}

During the initial six acquisition sessions each animal performed a total of 36 trials with a retention delay of $15 \mathrm{sec}$ (Fig. 10). Total correct scores were analyzed using an ANOVA that revealed a highly significant group effect $[F(4,46)=26.4, p<$ $0.0001]$. Subsequent Newman-Keuls tests showed that this reflected the poor performance of the Fx and CB groups, which were significantly worse than any of the other three groups $(p$ $<0.001)$. There were no other group differences.

All of the animals then performed a total of 60 trials, comprising 20 trials at each of three delays $(10,30$, and $60 \mathrm{sec})$. The mean percentage correct scores for the five groups are shown in Figure 10. An ANOVA revealed a highly significant group effect $[F(4,40)=9.66, p<0.001]$, a delay effect $[F(2,80)=13.8, p$ $<0 .(0) 1]$, and a group $\times$ delay interaction $[F(8,80)=2.21, p$ $<0.05]$. As with the acquisition data, the group effects reflected the poor performances of both the $\mathrm{Fx}$ and $\mathrm{CB}$ groups who dif fered significantly from all of the other groups $(p<0.01)$, but did not differ from each other. Likewise, the interaction reflected the abnormal pattern of scores produced by the Fx and CB groups, both of whom failed to show such clear delay effects as the other three groups. This was a consequence of the floor effects shown by subjects in each of the two groups. There were no wher group differences.

\section{Discussion}

The present study compared the effects of lesions in a number of outputs from the anterior thalamic nuclei on three tests of spatial memory. The DNMP task revealed a clear dissociation. as only the Fx and MPFc groups were impaired. The T-maze alternation task also produced clear group differences, but this time only the Fx and CB groups were impaired. This double dissociation highlights the different contributions of the medial prefrontal cortex and the cingulum bundle to spatial memory. Animals with extensive cytotoxic lesions of the cingulate and retrosplenial cortices were, however, unimpaired on both tasks. This latter finding implicates the cingulum bundle in the effects of cingulate cortex lesions.

Fornix and medial prefrontal lesions both produced similar disruptions of DNMP performance. Both groups suffered a loss of accuracy as measured by the signal detection indices SI and $\mathrm{A}^{\prime}$, although only the Fx group showed a delay-dependent effect. The Fx and MPFc groups also showed an increase in bias, as measured by $\mathrm{B}^{\prime \prime}$ and RI. The changes in these two bias indices, which are thought to measure slightly different aspects of bias ( $\mathrm{B}^{\prime \prime}$ - perceptual bias, and RI-a more general responsivity bias measure), were most pronounced in the MPFc group. Although these two groups also showed some minor changes in response latency, other groups showed similar changes even though they were unimpaired on the DNMP task. It would appear therefore that more nonspecific behavioural changes cannot account for the deficits shown by either the Fx or the MPFc groups.

An earlier study into the effects of aspiration lesions of the medial prefrontal cortex also found impairments on the similar delayed matching-to-position (DMP) task (Dunnett, 1990), but the pattern of results was different. While some rats showed delay-dependent deficits, others had such strong biases that they performed at chance at all delays $(0-24 \mathrm{sec})$. It was concluded that rostral medial prefrontal damage was sufficient to induce delay-dependent deficits, but that lesions involving the caudal prelimbic cortex could induce a more severe, nonspecific impairment (Dunnett, 1990). A further study showed that infusions of the muscarinic blocker, scopolamine. in the region of the prelimbic cortex disrupted DNMP performance (Dunnett et al., 1990). The disruption resembled that in the MPFc animals, as there was no interaction with delay but the rats were able to respond at close to $90 \%$ accuracy at the shortest delay (Dunnett et al., 1990).

It therefore appears that cellular damage or cholinergic blockade in the prelimbic cortex is sufficient to produce a mild DNMP deficit that largely stems from an increase in bias. This effect was found for all measures of bias, so it was not possible to ascribe it to a particular form of perseveration. None of the MPFc animals, however, displayed a bias as severe as that reported by Dunnett (1990), even though the lesions were at least as extensive. This suggests that the extreme bias observed by Dunnell (1990) was due to white matter damage in the region of the prelimbic cortex, leading to the disconnection of additional prefrontal tissue. The present study also confirmed that extensive cellular damage to the cingulate cortices does not affect DNMP performance (Neave et al., 1994). As lesions of the thalamic nucleus medialis dorsalis (MD) also spare DNMP performance (Neave et al., 1993), the key thalamic-frontal inputs are likely to be those from the anterior thalamic nuclei (Aggleton and Sahgal, 1993).

The second experiment, which involved lcarning a lever dis- 
crimination and then performing a series of reversals, also found evidence of a lesion dissociation. On this occasion the only significant differences were between the Fx and MPFc groups with the CINGc group. Thus, like Experiment 1, the results highlight the different contributions of the medial prefrontal and cingulate cortices to the performance of spatial tasks. Unlike previous studies, the Fx group did not show a clear deficit on the "reversal" sessions (Neave et al., 1994), but this may reflect the relatively small size of the group.

In the final task, T-maze alternation, only the $F x$ and $C B$ groups were impaired, while the MPFc and CINGc groups performed at a normal level. These findings appear to run counter to many previously published experiments. This is because lesions of the cingulate/retrosplenial cortices (Sutherland et al., 1988; Markowska et al., 1989; Sutherland and Rodriguez, 1989; Sutherland and Hoesing, 1993) and lesions of the medial prefrontal cortex (Brito et al., 1982; Thomas and Spafford, 1984; Brito and Brito, 1990; Shaw and Aggleton, 1993; Granon et al., 1994) are often sufficient to disrupt the type of spatial memory (allocentric) involved in the alternation task. These reports include comparable studies of T-maze alternation, many of which used retention delays similar to those in the current study (Thomas and Brito, 1980; Thomas and Spafford, 1984; Markowska et al., 1989; Brito and Brito, 1990; Shaw and Aggleton, 1993; Granon et al., 1994). As the CINGc lesions were as extensive as those that disrupt T-maze alternation (Markowska et al., 1989) and the MPFc lesions removed nearly all of the prelimbic area, which is often regarded as being critical (Brito et al., 1982; Thomas and Spafford, 1984), the present findings require explanation. Furthermore, unpublished studies have confirmed that the strain of rat used in the current research typically relies on allocentric cues to perform the T-maze alternation task.

The most plausible explanation for the apparent discrepancies in the literature concerns the impact of inadvertant damage to white matter. Most conventional cingulate lesions disrupt the cingulum bundle, but there is a tendency to overlook the potential impact of this damage because the bundle carries so many cingulate connections. By comparing the CINGc and $\mathrm{CB}$ groups it is evident that this assumption is incorrect for T-maze alternation. Other evidence for an independent cingulum bundle contribution comes from reports that neurotoxic lesions of either the anterior cingulate or retrosplenial cortex fail to affect $T$-maze alternation (Neave et al., 1994). A comparison between electrolytic and cytotoxic retrosplenial lesions has also emphasized the separate contribution of cingulum bundle damage to spatial memory (Meunier and Destrade, 1988). While the CINGc group show that damage to this cortex is not sufficent to impair this task, the deficit in the CB group may still reflect a combination of temporal and cingulate disconnections. Finally, the MPFc group shows that the critical cingulum connections are unlikely to arise from the medial prefrontal cortex, leaving the anterior thalamic nuclei as the most likely source.

The extent of the independent contribution from cingulum bundle damage is likely to be task specific. There is, for example, evidence that the disruptive effects of retrosplenial lesions on the Morris water maze are not due to cingulum bundle damage (Sutherland and Hoesing, 1993), as rats still perform poorly after a unilateral cytotoxic lesion and a contralateral aspiration lesion (Sutherland and Hoesing, 1993). This surgery did, however, cut the cingulum bundle in one hemisphere, and this may influence performance. Finally, the evidence that cingulum bundle damage can contribute to memory deficits may be of relevance when considering cases of human amnesia associated with retrosplenial damage (Valenstein et al., 1987).

The effects of conventional medial prefrontal lesions on tests of allocentric memory may also involve cingulum bundle disruption. While rostral cingulum bundle damage may increase frontal disconnection, more caudal damage may also disrupt other connections, including those from the anterior thalamic nuclei (Domesick, 1970). As a consequence, the caudal and ventral extent of a medial prefrontal lesion may considerably influence its effect. For example, the normal T-maze scores of the MPFc animals contrasted with an impairment following large aspiration lesions of the medial prefrontal cortex in rats tested under the same conditions (Shaw and Aggleton, 1993). Smaller medial prefrontal lesions resulted in only transient impairments (Shaw and Aggleton, 1993), as have been reported by other experimenters (Thomas and Brito, 1980; Thomas and Spafford, 1984). Thus, relatively mild, transient impairments on T-maze alternation may reflect both direct cellular damage and some prefrontal disconnection, while more severe deficits reflect additional disconnections of cortical and subcortical regions.

The present study indicates that different anterior thalamic outputs have qualitatively different contributions to spatial processes. While cingulum bundle lesions disrupted only T-maze alternations, medial prefrontal lesions only disrupted DNMP. The former result is paralelled by the finding that mamillary bodies lesions also disrupt T-maze alternation but not DNMP (Aggleton et al., 1990, 1991, 1995). These results suggest the existence of more than one hippocampal-diencephalic pathway involved in spatial memory. The T-maze task, which typically taxes allocentric memory, appears to involve a pathway from the hippocampus to the mamillary bodies, to the anterior thalamic nuclei, and then to some further target region via the cingulum bundle. Candidate regions include the subicular, entorhinal, and perirhinal cortices (Shihata, 1993), but not the anterior cingulate, retrosplenial, or medial prefrontal cortices.

In contrast, performance on the DNMP task, which taxes more egocentric or motorific cues, may involve a direct pathway from the hippocampus to the anterior thalamic nuclei, and from there to the medial prefrontal cortex. The involvement of the prefrontal cortex concurs with other lesion evidence pointing to the inportance of this region for egocentric task performance (Kesner et al., 1989; King and Corwin, 1992), while the hippocampal and anterior thalamic contributions may be linked with the presence of units responsive to head direction (Taube et al., 1990; Mizumori and Williams, 1993; Goodridge and Taube, 1994). Before concluding that the key difference between Experiments 1 and 3 concerns the involvement of egocentric versus allocentric memory it will be necessary to identify the spatial cues used to perform the DNMP task. It will also be necessary to examine other possible explanations. One concerns the fact that the animals were trained preoperatively on the DNMP task but not on the T-maze alternation task. With this in mind, we are currently testing animals with cingulum bundle lesions on acquisition of the DNMP task. Another relevant issue is whether the frontal lesion deficit principally arises from perseverative behaviour, so that performance of the DNMP task is more easily disrupted by such postoperative changes. This will be tested by examining the effects of cytotoxic lesions of the medial prefrontal cortex on allocentric tasks that are more sensitive to changes in bias than $\mathrm{T}$-maze alternation. 


\section{References}

Aggleton JP. Sahgal A (1993) The contribution of the anterior thalamic nuclei to anterograde memory. Neuropsychologia 31:1001--1019.

Aggleton JP, Hunt PR, Rawlins JNP (1986) The effects of hippocampal lesions upon spatial and non-spatial tests of working memory. Behav Brain Res 19:133-146.

Aggleton JP, Hunt PR, Shaw C (1990) The effects of mamillary body and combined amygdalar-fornix lesions on tests of delayed nonmatching-to-sample in the rat. Behav Brain Res 40:145-157.

Aggleton JP, Keith AB, Sahgal A (1991) Both fornix and anterior thalamic, but not mammillary, lesions disrupt delayed nonmatching-toposition memory in rats. Behav Brain Res 44:151-161.

Aggleton, JP. Keith AB, Rawlins JNP, Hunt PR, Sahgal A (1992) Removal of the hippocampus and transection of the fornix produce comparable deficits on delayed nonmatching to position by rats. Behav Brain Res 52:61-71.

Aggleton JP, Neave N, Nagle S, Hunt PR (1995) A comparison of the effects of anterior thalamic, mamillary body and fornix lesions on reinforced spatial alternation. Behav Brain Res 68:91-101.

Brito GNO, Brito LSO (1990) Septohippocampal system and the prelimbic sector of frontal cortex: a neuropsychological battery analysis in the rat. Behav Brain Res 36:127-146.

Brito GNO, Thomas GJ, Davis BJ, Gingold SI (1982) Prelimbic cortex, mediodorsal thalamus, septum, and delayed alternation in rats. Exp Brain Res 46:52-58.

Bruin JPC de, Sanchez-Santed F, Heinsbroek RPW, Donker A, Postmes $P$ (1994) A behavioural analysis of rats with damage to the medial prefrontal cortex using the morris water maze: evidence for behavoural flexibility, but not for impaired spatial navigation. Brain Res 323-333.

Conde F, Audinat E, Maire-Lepoivre E, Crepel F (1990) Afferent connections of the medial frontal cortex of the rat. A study using retrograde transport of fluorescent dyes. I. Thalamic afferents. Brain Res Bull 24:341-354.

Domesick VB (1970) The fasciculus cinguli in the rat. Brain Res 20 : $19-32$.

Dunnett SB (1990) Role of prefrontal cortex and striatal output systems in short-term memory deficits associated with ageing, basal forebrain lesions, and cholinergic rich grafts. Can J Psychol 44:210-232.

Dunnett SB, Wareham AT, Torres EM (1990) Cholinergic blockade in prefrontal cortex and hippocampus disrupts short-term memory in rats. Neuroreport 1:61-64.

Frey PW, Colliver JA (1973) Sensitivity and responsivity measures for discrimination learning. Learn Motiv 4:327-342.

Friedman HR, Janas JD, Goldman-Rakic PS (1990) Enhancement of metabolic activity in the diencephalon of monkeys performing working memory tasks: a 2-deoxyglucose study in behaving rhesus monkeys. J Cognit Neurosci 2:18-31.

Goodridge JP, Taube JS (1994) The effect of lesions of the postsubiculum on head direction cell firing in the anterior thalamic nuclei. Soc Neurosci Abstr 334.9.

Granon S, Vidal C, Thinus-Blanc C, Changeux J-P, Poucet B (1994) Working memory, response selection, and effortful processing in rats with medial prefrontal lesions. Behav Neurosci 108:883-891.

Groen T van, Wyss JM (1992) Connections of the retrosplenial dysgranular cortex in the rat. J Comp Neurol 315:200-216.

Horikawa K, Kinjo N, Stanley L C, Powell EW (1988) Topographic organization and collateralization of the projections of the anterior and laterodorsal thalamic nuclei to cingulate areas 24 and 29 in the rat. Neurosci Res 6:31-44.

Jay TM, Glowinski J, Thierry AM (1989) Selectivity of the hippocampal connection to the prelimbic area of the prefrontal cortex in the rat. Brain Res 505:337-340.

Kesner RP, Farnsworth G, DiMattia BV (1989) Double dissociation of egocentric and allocentric space following medial prefrontal and parietal cortex lesions in the rat. Behav Neurosci 103:956-961.

King VR, Corwin JV (1992) Spatial deficits and hemisperic asymmetries in the rat following unilateral and bilateral lesions of posterior parietal or medial agranular cortex. Behav Brain Res 50:53-68.

Kolb B (1984) Functions of the frontal cortex of the rat: a comparative review. Brain Res Rev 8:65-98.

Markowska AL, OIton DS, Murray EA, Gaffan D (1989) A comparative analysis of the role of fornix and cingulate cortex in memory: rats. Exp Brain Res 74:187-201.

Meunier M, Destrade C (1988) Electrolytic but not ibotenic acid lesions of the posterior cingulate cortex produce transitory facilitation of learning in mice. Behav Brain Res 27:161-172.

Mizumori SJY, Williams JD (1993) Directionally selective mnemonic properties of neurons in the lateral dorsal nucleus of the thalamus of rats. J Neurosci 13:4014-4028.

Mufson EJ, Pandya DP (1984) Some observations on the course and composition of the cingulum bundle in the rhesus monkey. J Comp Neurol 225:31-43.

Neave N, Sahgal A, Aggleton JP (1993) Lack of effect of dorsomedial thalamic lesions on automated tests of spatial memory in the rat. Behav Brain Res 55:39-49.

Neave N, Lloyd S, Sahgal A, Aggleton JP (1994) Lack of effect of lesions in the anterior cingulate cortex and retrosplenial cortex on certain tests of spatial memory in the rat. Behav Brain Res 65:89101

Sahgal A (1983) Vasopressin retards the acquisition of positively reinforecd lever pressing in homozygous Brattelboro rats. Regul Pept $5: 317-326$

Sahgal A (1987) Some limitations of indices derived from signal detection theory: evaluation of an alternative index for measuring bias in memory tasks. Psychopharmacology (Berlin) 91:517-520.

Shaw C, Aggleton JP (1993) The effects of fornix and medial prefrontal lesions on delayed non-matching-to-sample by rats. Behav Brain Res 54:91-102.

Shibata H (1993) Efferent projections from the anterior thalamic nuclei to the cingulate cortex in the rat. J Comp Neurol 330:533-542.

Sutherland RJ, Hoesing JM (1993) Posterior cingulate cortex and spatial memory: a microlimnology analysis. In: Neurobiology of cingulate cortex and limbic thalamus: a comprehensive treatise (Vogt BA, Gabriel M, eds), pp 461-477. Boston: Birkhauser.

Sutherland RJ, Rodriguez AJ (1989) The role of the fornix/fimbria and some related subcortical structures in place learning and memory. Behav Brain Res 32:265-277

Sutherland RJ, Whishaw IQ, Kolb B (1988) Contributions of cingulate cortex to two forms of spatial learning and memory. $J$ Neurosci 8:1863-1872.

Swanson LW (1992) Brain maps: structure of the rat brain. Amsterdam: Elsevier.

Taube S, Muller RU, Ranck JB (1990) Head direction cells recorded from the postsubiculum in freely moving rats. 1. Description and quantitative analysis. J Neurosci 10:420-435.

Thomas GJ, Brito GNO (1980) Recovery of delayed alternation in rats after lesions in medial frontal cortex and septum. J Comp Physiol Psychol 94:808-818.

Thomas GJ, Spafford PS (1984) Deficits for representational memory induced by septal and cortical lesions (singly and combined) in rats. Behav Neurosci 98:394-404.

Valenstein E, Bowers D, Verfaellie M, Heilman KM, Day A, Watson RT (1987) Retrosplenial amnesia. Brain 110:1631-1646.

Winer BJ (1971) Statistical principles in experimental design (2nd ed). Tokyo: McGraw-Hill.

Zeng D, Stuesse SL (1993) Topographic organization of efferent projections of medial frontal cortex. Brain Res Bull 32:195-200. 